Research Article

\title{
Load Characteristics and Modeling Methods for the Flow Regulator of a Solid Ducted Rocket
}

\author{
An Wang $\mathbb{D}^{1,2}$ and Qinghua Zeng $\mathbb{D}^{2}$ \\ ${ }^{1}$ College of Aerospace Science and Engineering, National University of Defense Technology, Changsha, Hunan 410073, China \\ ${ }^{2}$ School of Aeronautics and Astronautics, Sun Yat-Sen University, Guangzhou, Guangdong 510006, China \\ Correspondence should be addressed to Qinghua Zeng; zqinghua@sysu.edu.cn
}

Received 26 July 2019; Accepted 22 October 2019; Published 18 November 2019

Academic Editor: Rosario Pecora

Copyright ( 2019 An Wang and Qinghua Zeng. This is an open access article distributed under the Creative Commons Attribution License, which permits unrestricted use, distribution, and reproduction in any medium, provided the original work is properly cited.

\begin{abstract}
The load characteristics for the flow regulator of a solid ducted rocket are discussed in this paper. The mechanism and the influence factor of the load in the flow regulator were studied both theoretically and experimentally, and the system load and the working load were divided according to the mechanism. Additionally, the load influence on the working quality of the flow regulator and the ducted rocket were analyzed. System modeling of the flow regulator was carried out based on the working mechanism of the gas generator and the Stribeck friction model, and models of the actuator, gas generator, and load were built. Furthermore, considering the identification of the model parameters and the inapplicability of the conventional Stribeck identification method to the flow regulator, a new Stribeck model parameter identification method was proposed. A sine wave was used as the signal source, and specially designed devices were used to accomplish the switch of the working condition. Through the use of the genetic algorithm, the rotational inertia and parameters of the system load and working load were identified by stages. Finally, the validation of the identification method was carried out with a simulation.
\end{abstract}

\section{Introduction}

A solid ducted rocket is a propulsion device developed based on solid rocket and ramjet technology, possessing the advantages of high specific impulse, long range, small size, low weight, compact structure, and so on. It is an optimal propulsion device for the new generation of aircraft in aerospace [1-4].

The gas flow is designed based on the predetermined trajectory for a solid ducted rocket in the early stage, usually realized in the form of a prefabricated propellant grain structure or a burning rate preconfiguration because an active regulator cannot be used in this form. The trajectory of the aircraft using this engine will be restricted severely due to the reason above, and the performance of the ducted rocket will decrease rapidly once the flight condition deviates from the design condition. Due to the existence of a variety of disturbances during a real flight and the increasing complexity of flight missions, the active control technology of flow regulation has become the key focus of ducted rocket research.
Generally, a pressure sensitive propellant is used in a choked solid ducted rocket. The flow production rate can be adjusted by changing the nozzle throat area using a regulator valve. This is one of the most powerful regulation methods at present. However, the working environment of a flow regulator device is extremely harsh, and the problems of thermal protection, gas tightness, valve fit in the throat, solid sediment clogging, actuator loads, and so on need to be solved urgently.

The actuator load is the primary factor that influences the control effect of flow regulation. As a typical mechanical servo system, friction is the primary reason that causes the load in a flow regulator. Friction is a complicated physical phenomenon with nonlinear and uncertain characteristics, and it leads to steady state errors, stick slips, limit cycles, and other phenomena that degrade the performance of a servo system, especially in the low speed range of a servo system [5].

In-depth research has been carried out by scholars in various fields in order to understand the friction phenomenon 
correctly. Previous research achievements regarding friction can be summarized as belonging to the following categories: static friction, Coulomb friction, viscous friction, Stribeck effect, frictional memory, and stick slip motion [6-9]. The modeling of friction plays an important role in analyzing and solving the phenomenon caused by friction, and static modeling and dynamic modeling are divided according to whether a differential equation is used to model the friction. Among these models, the Stribeck model is a typical static model. It was proposed by the scholar Stribeck on the basis of previous studies and his own experiments, and it describes four lubrication stages that a system goes through at low speed: static friction, boundary lubrication, partial fluid lubrication, and full fluid lubrication. A Stribeck friction model is proposed with four parameters. Another typical dynamic model called the LuGre friction model was used by the scholar Canudas [10]. The bristle model is used to describe friction; it contains four static parameters that are the same as those of the Stribeck model and two other dynamic parameters. It can accurately explain phenomena such as frictional memory, varying static friction, and presliding displacement.

For different friction models, the more parameters they possess, the more accurate the models will be, but the more difficult the parameter identification will be. To solve the problem related to friction, choosing a suitable model and identifying the method according to the system features is critical. Tan et al. proposed a LuGre model identification method based on the analysis of the steady state error [11]. The tracking accuracy of the servo system could be improved through friction compensation based on the proposed model. The recursive equations of the LuGre model were solved, and the problem of effectively detecting mane microdisplacement was discussed by Guo et al. [12]. Shao proposed a new modified LuGre model suited to a high-speed mechanical system with friction as well as an improved artificial fish swarm algorithm (IAFSA) method that introduced the chaotic search and Gauss mutation operators into a traditional artificial fish swarm algorithm in order to identify the parameters in the proposed modified LuGre friction model [13]. The angular speed over the zero phenomenon occurring in the angular speed free reduction was used to acquire the microdisplacement of the mane in reference [14]. The Stribeck curve was plotted according to the velocity and friction, and then a genetic algorithm was used to acquire the model parameter basis on the Stribeck curve in reference [15].

The load characteristics of a flow regulator have a profound influence on the working quality of a ducted rocket. However, as far as can be determined, the friction and the load it brings to the flow regulator have rarely been studied except in reference [16], where the friction generated in the gas flow regulation of ducted rockets was considered, and a compensation method was proposed and proved with a simulation result. However, this method only considered the viscous friction without taking other friction forces into account, and the mechanism and characteristics of the friction in the flow regulator were not discussed in detail. The way the fuel inject into the combustor in solid ducted rocket is one of the maximum differences in comparison with liquid
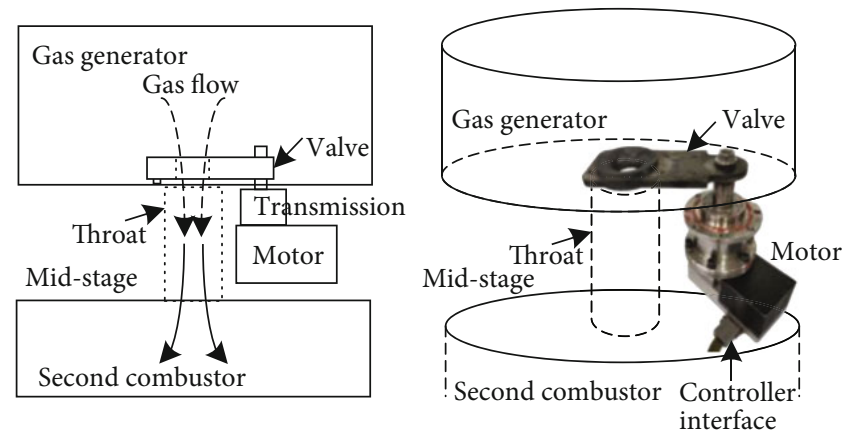

Figure 1: Schematics of the gas generator and the flow regulator.

ramjets $[17,18]$ that makes the load characteristics carried out in this paper can only be applied to solid ducted rocket.

To make up for the deficiencies discussed above, research on the load in the flow regulator during the working process of a ducted rocket was carried out in this study. The influences of the load on the working quality of the flow regulator and the ducted rocket were analyzed, and the load model of the flow regulator was built. This laid a foundation for the research on the ducted rocket control.

The rest of this paper is organized as follows. First, the characteristics of the load in a flow regulator and the influence factors are given. Then, the load model of a gas generator is built. Finally, a new parameter identification method for the Stribeck model is proposed and verified in a simulation case.

\section{Analysis of the Load Characteristics in a Flow Regulator}

2.1. Definition of a Flow Regulator. The flow regulator discussed in this paper regulates gas flow with a gate valve, which is a valve placed above the throat that changes the throat area by rotational motion and that has a good transmission performance, gas tightness, adiabaticity, and a compact structure. However, the valve is under a heavy impact load due to the high-speed gas flow working environment, so it will inevitably influence the performance of flow regulation and ducted rocket control. The compositions of the gas generator and the flow regulator are shown in Figure 1. It is important to research the load characteristics of a flow regulator because the flow regulator benefits the control of a ducted rocket and the completion of a flight mission.

2.2. Load Mechanism and Factors. DC motors are widely used as the actuators in flow regulator devices to complete gas flow regulation because they are simple and reliable, possessing the advantages of good manufacturability and convenience of use and maintenance, and they can easily accomplish the energy management of an aircraft. A motor needs to include a reducer, a transmission system, and other mechanical devices to convert the motor rotation into the movement of a valve. This process inevitably produces friction. As shown in Figure 2, when the flow regulator followed a low speed ramp signal in a nonworking condition, the system exhibited a stick slip and steady state error phenomenon. 

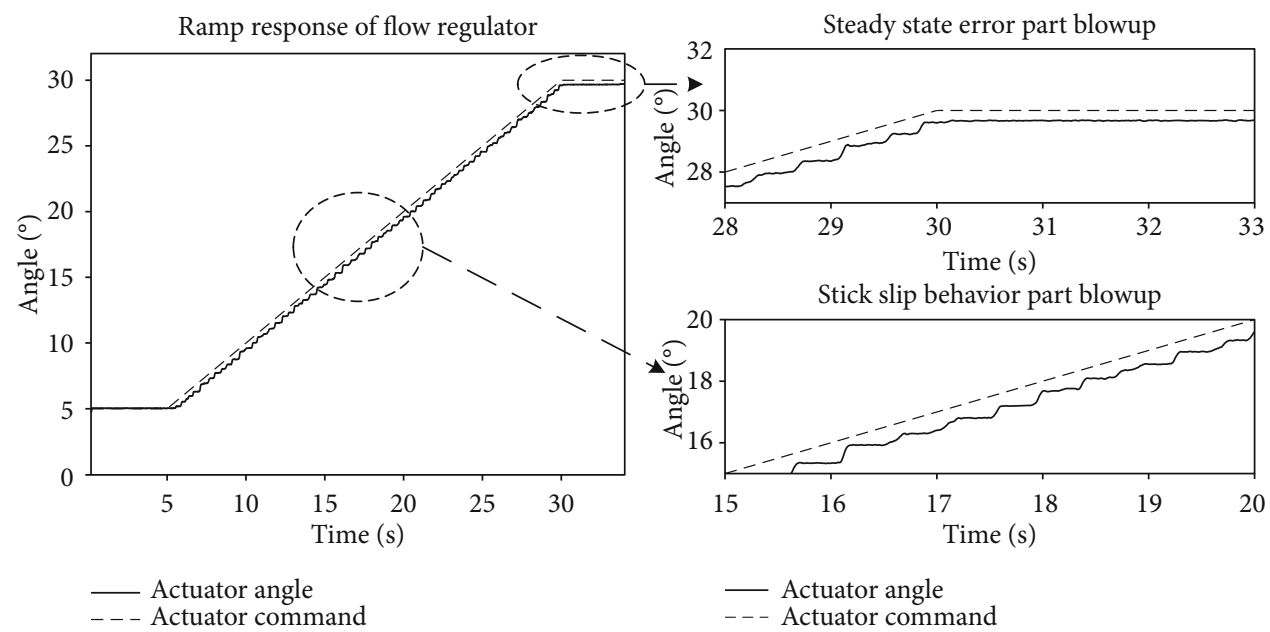
- - Actuator command

- Actuator angle
-- Actuator command

Figure 2: Stick slip behavior and steady state error of the flow regulator.

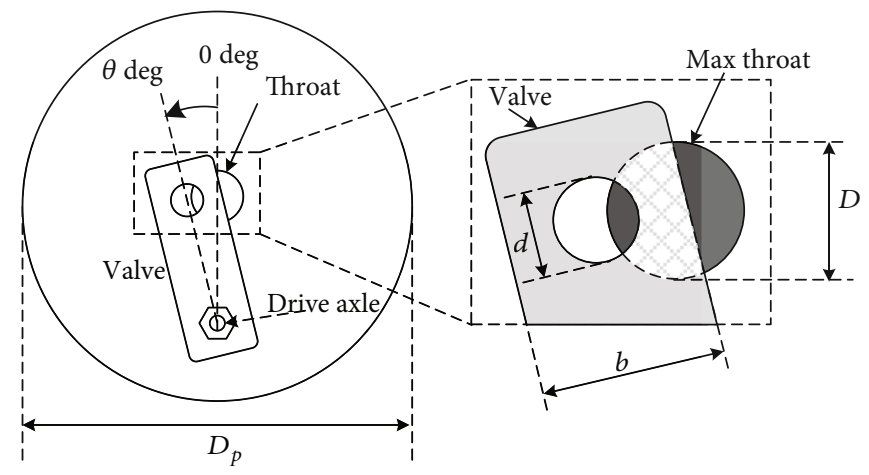

Impacted area

Effective throat area

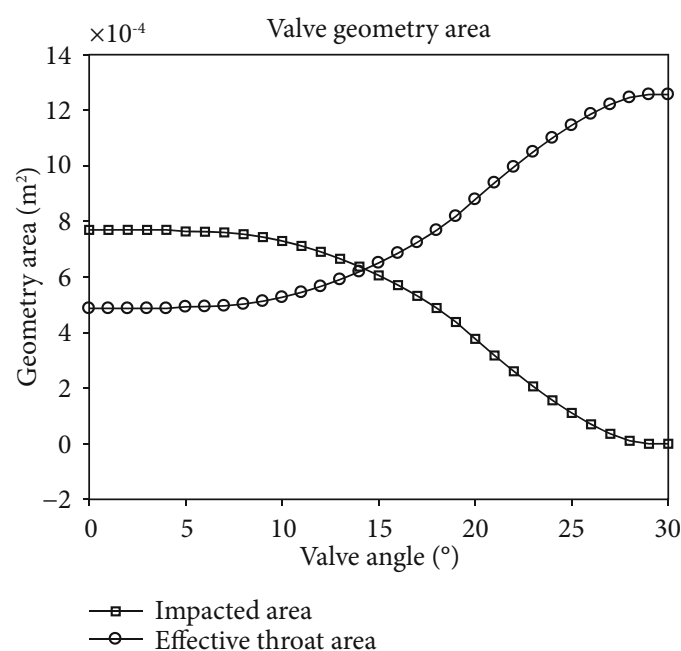

FIgURe 3: Platform of the gas generator and schematic of the impact area.

This shows that the flow regulator device had the friction torque that exists in most mechanical devices when a gas generator is in a nonworking condition. This type of friction is only related to the mechanical structure, and it is not affected by the working state of the gas generator; it is defined as the system load of the flow regulator in this paper.

However, when a gas generator is working, high-speed gas flow is generated in the gas generator, and this flow enters the second combustor through the valve. This will produce a huge impact load to the valve, making the valve encounter the bottom of the gas generator. Contact pressure also exists, which produces friction torque that impedes the valve movement; this is the main part of the flow regulator load, and it is defined as the working load of the flow regulator in this paper.

As shown in reference [19], the friction in the contact surface is related to the contact pressure, and the contact pressure between the valve and the gas generator bottom is related to the pressure of the gas generator, the pressure of the second combustor, the impact area, and other influence factors.

In the ducted rocket ground experiment, the valve was a rectangular gate valve with a circular flow hole and the circular hole was the minimum throat area of the gas generator when the valve was at the position of 0 degrees. As shown in Figure 3, the maximum throat was equal to the sum of the effective throat area and the impacted area.

The gas generator and regulator geometry parameters in Figure 3 are as follows: the minimum diameter $d=25 \mathrm{~mm}$, the maximum diameter $D=40 \mathrm{~mm}$, the valve width $b=54 \mathrm{~mm}$, and the diameter of the gas generator $D_{P}=350 \mathrm{~mm}$, which is also the diameter of the propellant. The curve of the impacted area and effective throat area versus the valve angle can be obtained according to the parameter above.

The regulator valve was turned from 0 degrees to 30 degrees at a velocity of 50 degrees/s when the pressure of the gas generator was in a stable state. In this process, the 

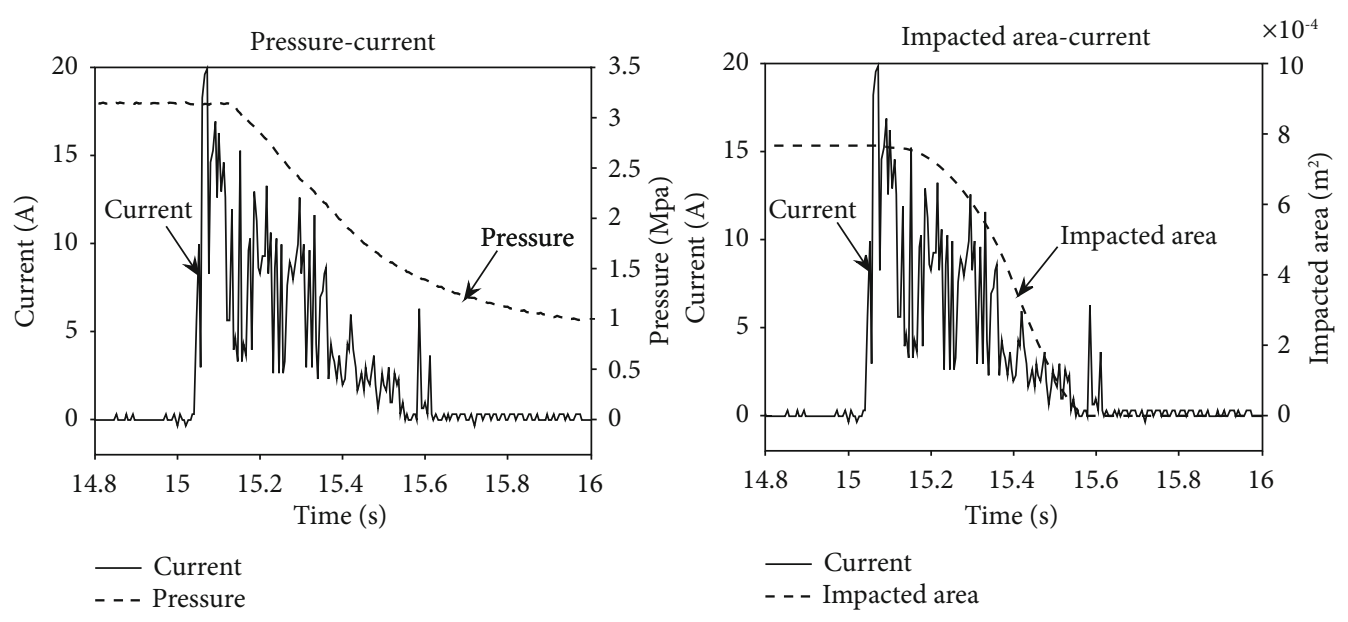

Figure 4: Motor current decrease with the decrease of the pressure and the impact area.

effective throat area increased with the decrease of the impacted area. Otherwise, the pressure of the gas generator decreased with the decrease of the choked level, which was caused by the increase of the effective throat.

Because of the direct relationship between the motor current and the output torque, when the valve was moving at an approximately uniform speed, the load torque could be determined from the motor current. In the experiment above, the motor current and the pressure of the gas generator were measured and the impacted area was calculated by the motor angle. Later, the curve in Figure 4 was obtained. The results of the experiment show that the motor current decreased with the decrease of the impacted area and the pressure of the gas generator.

The results above qualitatively verified that the working load was related not only to the characteristics of the contact surface but also to the impacted area and the pressure of the gas generator. The impacted area and the pressure of the gas generator were also affected by the control process of flow regulation. In addition, the working load of the flow regulator had a direct influence on the process of control, which means there was a coupling relationship between the working load and the control process.

In conclusion, the load of a flow regulator can be divided into two parts: the system load and the working load. Both of the loads possess the same complexity, nonlinearity, and uncertainty characteristics as the friction. In addition, a coupling relationship with the control process exists. The friction characteristics of the loads bring stick slips, steady state errors, and other phenomena to the system that will cause an unsatisfactory signal follow result and the increase of control error. The thrust control and the flight mission will also be affected. Furthermore, if the control strategy in the aircraft does not handle the load well, the power consumption of the flow regulator will increase rapidly, the energy onboard will be severely depleted, and the working time of the flow regulator will be greatly reduced. At the same time, other electrical equipment onboard will be affected if the centralized power supply is used in the aircraft, which will lead to a failure of the flight mission. To solve the various problems that the load of the flow regulator brings, research on the load must be carried out.

\section{Load Modeling of the Flow Regulator}

According to the analysis of the load mechanism in the last section and the system loop of the flow regulator, the system block of the flow regulator control system including the load model is shown in Figure 5.

The modeling processes of the DC motor, gas generator, and load are discussed below in detail.

3.1. Modeling of the DC Motor. According to the schematic of the DC motor in a flow regulator, which is shown in Figure 6, and taking the friction and the load characteristics into account, the rotation dynamics equation of a flow regulator can be built as shown in

$$
\begin{aligned}
R_{a} I_{a}+L_{a} \frac{d I_{a}}{d t} & =U_{a}-K_{e} \dot{\theta} \\
J \frac{d \dot{\theta}}{d t} & =K_{m} I_{a}-M_{L}-M_{f} .
\end{aligned}
$$

According to the mathematical model above, the system block of the motor, including the system and the working load, can be built as shown in Figure 7 .

3.2. Modeling of the Gas Generator. Several assumptions were set as follows before the model of the gas generator was built:

(1) The solid propellant was end burning, and the burning area remained unchanged

(2) The gas parameters were the same in every area of the gas generator

(3) The gas obeyed the ideal gas state equation

(4) The gas temperature in the gas generator remained unchanged 


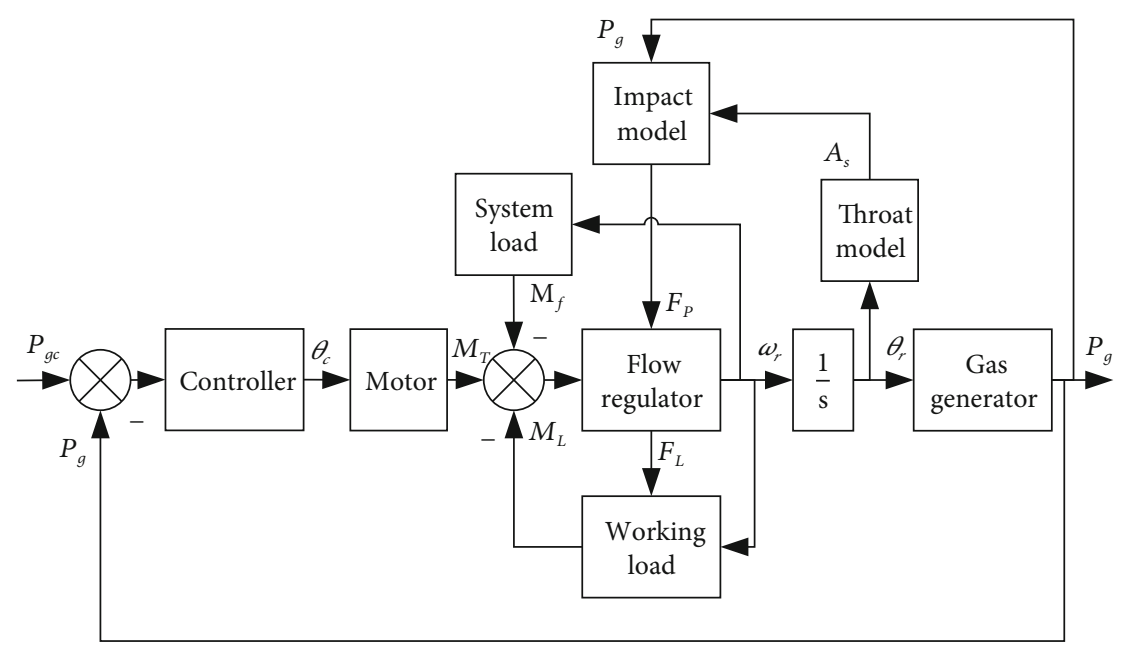

FIGURE 5: System block diagram of the flow regulator including the load model.

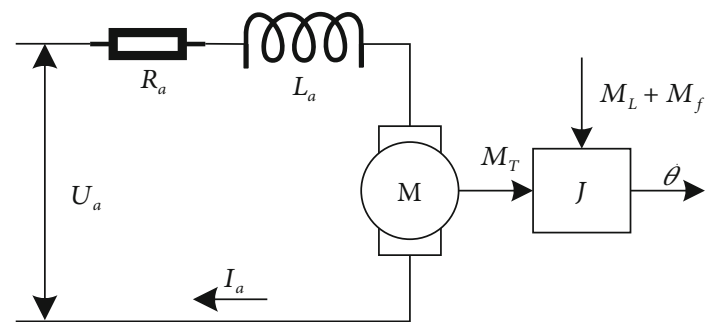

Figure 6: Schematic of the DC motor in a flow regulator.

(5) The burning rate was the same in every section of the burning area

The converting rate of solid propellant is given as

$$
\dot{m}_{r}=\rho_{b} A_{b} r=\rho_{b} A_{b} a \rho_{g}{ }^{n}
$$

The gas flow rate through the valve is given as

$$
\dot{m}_{g}=\frac{p_{g} A_{t}}{C_{r}}
$$

According to the conservation of mass, the converting rate of solid propellant is equal to the sum of the gas flow rate through the valve and the mass rate of the gas in the free volume:

$$
\dot{m}_{r}=\dot{m}_{g}+\frac{d}{d t}\left(\rho_{g} V\right)=\dot{m}_{g}+V \frac{d \rho_{g}}{d t}+\rho_{g} \frac{d V}{d t} .
$$

The free volume is defined as the volume between the burning area and the throat, so the free volume of a gas generator can be written as $V(t)=V_{0}+\int_{0}^{t} A_{b} r \cdot d t$.
According to equations (2) and (3), and the ideal gas state equation

$$
\rho_{g}=\frac{p_{g}}{R_{g} T_{g}}
$$

equation (4) can be rewritten as

$$
\rho_{b} A_{b} a p_{g}{ }^{n}=\frac{p_{g} A_{t}}{C_{r}}+\frac{V}{R_{g} T_{g}} \frac{d p_{g}}{d t}+\frac{p_{g}}{R_{g} T_{g}} A_{b} r .
$$

Additionally, equation (6) can be written in the following form:

$$
\frac{V}{R_{g} T_{g}} \frac{d p_{g}}{d t}=\left(\rho_{b}-\rho_{g}\right) A_{b} a p_{g}{ }^{n}-\frac{p_{g} A_{t}}{C_{r}} .
$$

Due to the large difference between the density of a gas and a solid, $\rho_{b}>>\rho_{g}$, equation (7) can be simplified as

$$
\frac{d p_{g}}{d t}=\frac{R_{g} T_{g}}{V}\left(\rho_{b} A_{b} a p_{g}{ }^{n}-\frac{p_{g} A_{t}}{C_{r}}\right) .
$$

From the equations above, it is obvious that the pressure of the gas generator $p_{g}$ is a function of the throat area $A_{t}$, and $A_{t}$ is a function of the controlled quantity, the valve angle $\theta_{r}$. The propellant parameters in this paper are as follows: propellant density $\rho_{b}=1630 \mathrm{~kg} / \mathrm{m}^{3}$, propellant area $A_{b}=\pi D_{P}^{2} / 4$, burning rate coefficient $a=6.3$, and pressure exponent of burning rate $n=0.53$.

3.3. Modeling of the Load. Before building the load model of the gas generator, the following assumptions were made:

(1) The impact of the high-speed flow was concentrated at a point on the valve 


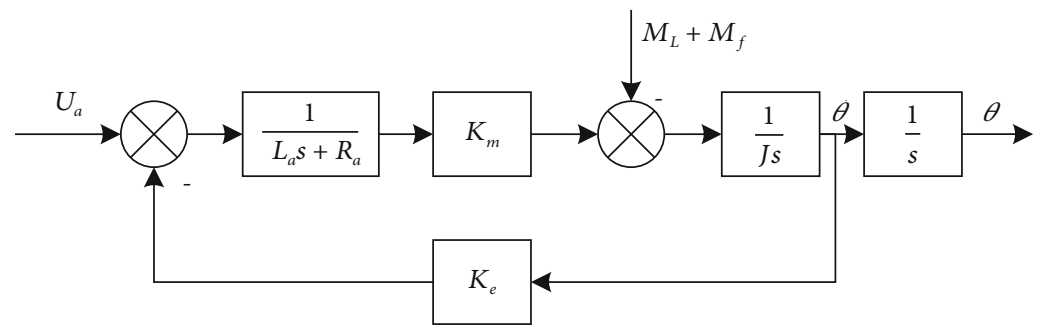

FIGURE 7: System block diagram of the motor including the system and the working load torque.

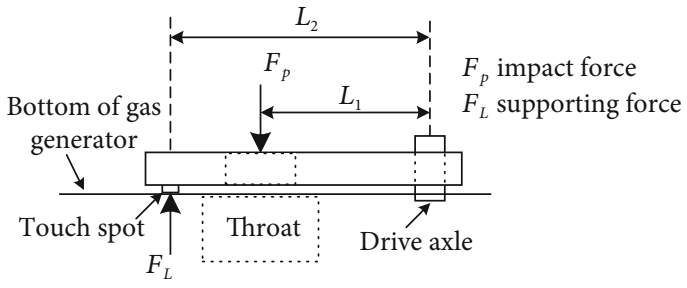

FIGURE 8: Force analysis of the flow regulator.

(2) The impact force $F_{P}$ was equal to the product of the impacted area $A_{s}$ and the pressure difference between the gas generator and the second combustor $p_{g}-p_{c 0}$

(3) There was a touch spot between the valve and the bottom of the gas generator, as shown in Figure 8

(4) There was no gap between the touch spot and the bottom of the gas generator in the nonworking condition, and the valve did not deform under the impact load

(5) There was no mechanical clearance in the flow regulator, and the motor angle $\theta$ and the valve angle $\theta_{r}$ satisfied the equation $\theta=i \cdot \theta_{r}$

(6) The combustion products of the solid propellant inevitably contain solid particles, such as metallic oxide; however, it takes a very small mass part of the combustion products; in addition, it is always in a low speed compared to the gaseous product. It means the solid products have low momentum, and the impact force due to the solid products can be ignored in assumption

As discussed above, the load torque of the flow regulator was divided into two parts: the system load torque $M_{f}$ and the working load torque $M_{L}$.

The system load torque was composed of the friction torque, and the friction model could be built based on the Stribeck model. The mathematical description of the system load torque is

$$
M_{f}=\operatorname{sgn}\left(\omega_{r}\right)\left(M_{c}+\left(M_{s}-M_{c}\right) e^{-\left(\omega_{r} / \omega_{s f}\right)^{\alpha}}\right)+\sigma_{2} \omega_{r} .
$$

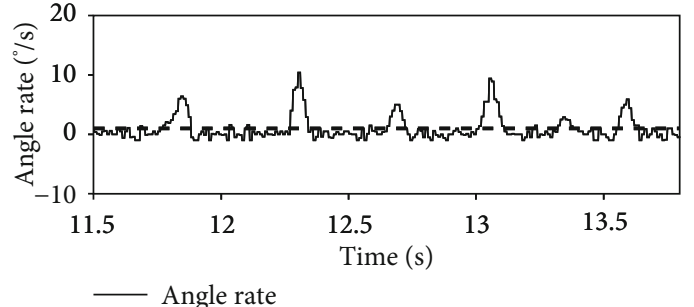

- - - Command

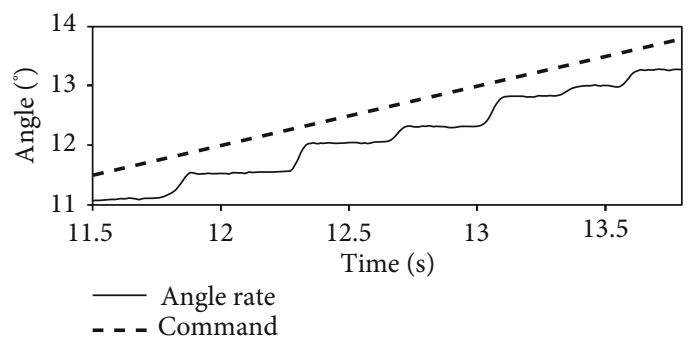

Figure 9: The flow regulator could not easily achieve uniform motion due to the stick slip.

According to the assumptions and the analysis above, the impact force can be written as

$$
F_{P}=\left(p_{g}\left(\theta_{r}, t\right)-p_{c 0}\right) \cdot A_{s}\left(\theta_{r}\right)
$$

where the impacted area $A_{s}$ is a function of the valve angle $\theta_{r}$.

The force analysis of the impact force and the supporting force is shown in Figure 8. The supporting force is equal to the contact pressure, and it can be written as follows according to the static equilibrium equation:

$$
F_{L}=F_{P} \cdot \frac{L_{1}}{L_{2}} .
$$

According to reference [19], which presented research on the load and the Stribeck model, we could build an extended Stribeck friction model to describe the working load torque. Its mathematical description is given as

$$
M_{L}=L_{2} \cdot F_{L} \cdot m_{L}\left(\mu_{c}, \mu_{s}, \omega_{s L}, \mu_{2}\right),
$$

$m_{L}=\left(\mu_{c}+\left(\mu_{s}-\mu_{c}\right) \cdot e^{-\left(\omega_{r} / \omega_{s L}\right)^{\alpha}}+\mu_{2} \cdot \omega_{r}\right)$. 

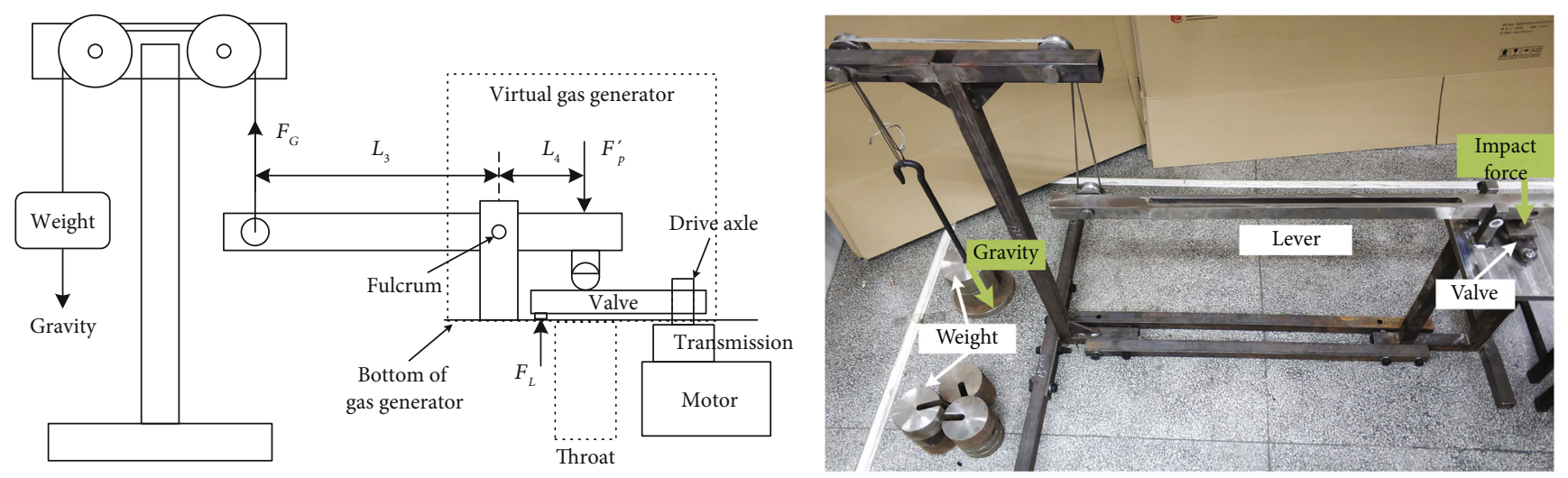

FIgURE 10: Working load simulation device for a gas generator.

Substituting equations (10) and (11) into equation (12), equation (12) can be rewritten as

$$
\begin{aligned}
M_{L}= & \left(p_{g}\left(\theta_{r}, t\right)-p_{c 0}\right) \cdot f_{A}\left(\theta_{r}\right) \cdot L_{1} \\
& \cdot\left(\mu_{c}+\left(\mu_{s}-\mu_{c}\right) \cdot e^{-\left(\omega_{r} / \omega_{s L}\right)^{2}}+\mu_{2} \cdot \omega_{r}\right) .
\end{aligned}
$$

Equations (9) and (13) are the mathematical descriptions of the system load and working load for the flow regulator. The next step of modeling is the identification of the model parameter.

\section{Model Parameter Identification}

For the parameter identification of the Stribeck friction model, the widely used method involves measuring the friction torque and the velocity when the system is moving at a uniform speed. Then, a Stribeck curve can be plotted using a system identification method on the curve, and the parameters of the Stribeck model can be acquired.

Furthermore, the primary factors for using the method above are a uniform motion mode and a speed control loop in the system, usually used in high-precision electromechanical devices. However, the research object in this paper does not possess the two factors above since the devices are used in the onboard and position motion modes often enough to achieve flow regulation. Therefore, the uniform motion mode and the speed control loop were unnecessary and would bring complexity and unreliability to the system used in aerospace. However, using a position motion mode to follow a uniform motion command was difficult to achieve due to the disturbance of friction, so plotting the Stribeck curve and identifying the parameter was almost impossible in this case. As shown in Figure 9, due to the stick slip phenomenon brought by friction, uniform motion was difficult to achieve using the motor in the flow regulator.

4.1. Identification Method. The identification method based on uniform motion used a special form to simplify the prob-

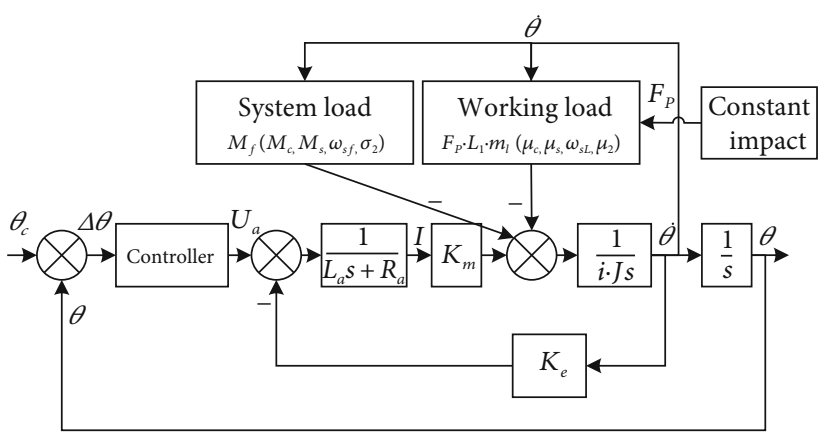

Figure 11: Simulation model of the flow regulator.

lem. For uniform motion, the rotation dynamic function in equation (1) can be simplified as

$$
J \dot{\omega}=M_{T}-M_{f}(\omega)=0 .
$$

There are two benefits to this special form. First, the friction torque that is difficult to measure is equal to the motor torque that is easy to estimate from the motor current. Second, the rotational inertia that is difficult to obtain in a complicated electromechanical system is not used in this function.

In fact, the special form of the rotation dynamic function can be used to identify the parameter in equation (14) when uniform motion is difficult to achieve. Further, the general form of the rotation dynamic function can be used, and the rotational inertia can be taken as an unknown parameter to be identified with the friction model parameter. Due to the basics of the general functions of the dynamics, this method does not rely on the forms of motion. However, the change range of the velocity should cover the common-use range of the system in order to acquire the appropriate sample data.

According to the modeling result of the previous section, the rotation dynamic function of the flow regulator including the friction load can be written as

$$
\begin{aligned}
J \cdot \dot{\omega}= & K_{m} \cdot I_{a}-F_{P} \cdot L_{1} \cdot m_{L}\left(\mu_{c}, \mu_{s}, \omega_{s L}, \mu_{2}\right) \\
& -M_{f}\left(M_{c}, M_{s}, \omega_{s f}, \sigma_{2}\right),
\end{aligned}
$$



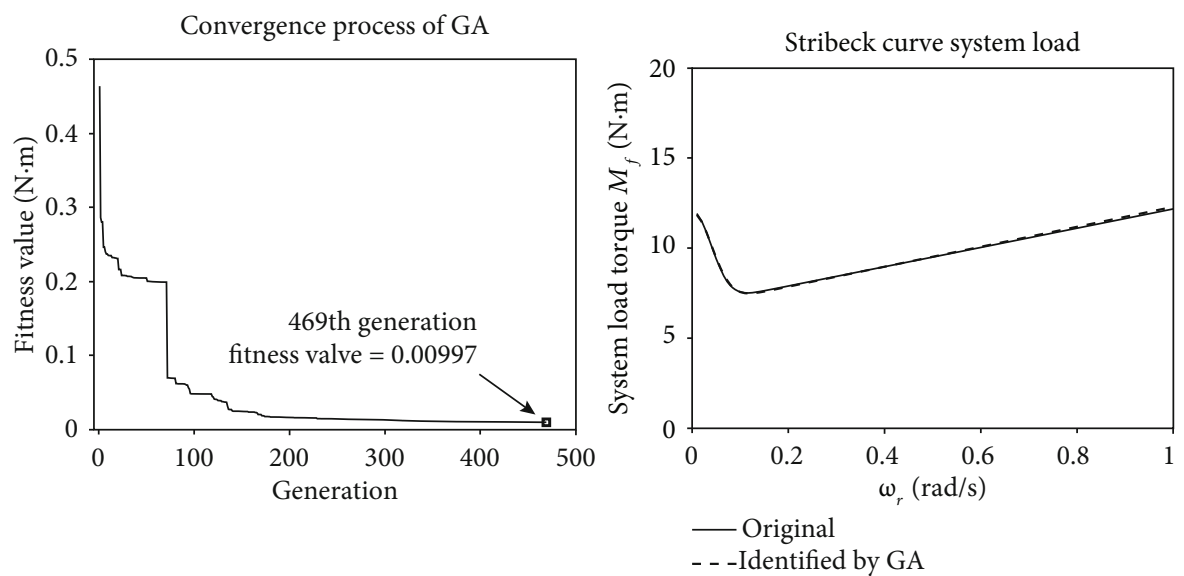

FIGURE 12: Parameter identification result of the system load model using the genetic algorithm.

where the parameters within brackets are the eight parameters to be identified in the load model, and the rotational inertia is another parameter to be identified in the system model.

The identification process was done in two steps. First, the four parameters in the system load and the rotational inertia in the nonworking mode were identified, and because the impact force was zero, equation (15) could be simplified as

$$
J \cdot \dot{\omega}=K_{m} \cdot I_{a}-M_{f}\left(M_{c}, M_{s}, \omega_{s f}, \sigma_{2}\right) .
$$

Then, a sine wave was used as a command signal, and the response signals of the angle rates of the valve and the motor current were measured, and the five parameters could be identified using a genetic algorithm. Second, after the five parameters above were identified, the known impact load was brought in to simulate the working condition, and the remaining four parameters could be identified in the same way.

The identification method could be achieved using the experimental device in Figure 10. This device could simulate the nonworking mode and the working mode in different conditions by adjusting the weights in the devices.

The weights of the devices can be adjusting as needed, and the simulation impact force $F_{P}^{\prime}$ can be adjusted as well; $F_{P}^{\prime}$ is the simulation force of $F_{P}$ in Figure 8. According to the schematic in Figure 10, the relation between impact force and weight can be written as follows:

$$
F_{P}^{\prime}=\frac{L_{3}}{L_{4}} \cdot m_{w} g
$$

where $m_{w}$ is the mass of the weight, which can be adjusted as needed, and the leverage $L_{3} / L_{4}$ in this device is 10 . Using the devices above, different working modes can be simulated in different $m_{w}$, and the parameter identification could be achieved in stages.

4.2. Verification. To verify the correctness of the method discussed in the previous section, the values of the nine under- identified parameters were given initially, and the valve angle, angle rate, and motor current were measured with a simulation. Using the measured data to reverse-engineer the identities of the model parameters, the difference between the reverse-engineered parameter and the given parameter before the simulation could be obtained, and the correctness of the identification method could be verified.

First, the simulation model of the flow regulator was built. This model is shown in Figure 11.

The system values were $L_{a}=0.00132 \mathrm{H}, R_{a}=1.56 \Omega$, $K_{m}=0.03 \mathrm{~N} \cdot \mathrm{m} / \mathrm{A}, K_{e}=0.02588 \mathrm{~V} / \mathrm{rad} / \mathrm{s}$, and $L_{1}=0.095 \mathrm{~m}$, and the nine underidentified parameters were $M_{c}=6.8250$ $\mathrm{N} \cdot \mathrm{m}, M_{\mathrm{s}}=12.025 \mathrm{~N} \cdot \mathrm{m}, \omega_{\mathrm{sf}}=0.0557 \mathrm{rad} / \mathrm{s}, \sigma_{2}=5.3462 \mathrm{~N} \cdot$ $\mathrm{m} \cdot \mathrm{s} / \mathrm{rad}, \quad \mu_{c}=0.052, \quad \mu=0.086, \quad \omega_{s L}=0.0326 \mathrm{rad} / \mathrm{s}, \quad \mu_{2}=$ 0.032 , and $J=0.000204 \mathrm{~kg} \cdot \mathrm{m}^{2}$.

4.2.1. Case 1: $F_{P}=0 N$ and $\theta_{c}=20 \cdot \sin (0.4 \pi r t)$. The parameter array to be identified was defined as $x=\left[M_{c} M_{s} \omega_{s f} \sigma_{2} J\right]$, and the genetic algorithm initial configuration was set up as follows: maximum generations $=1000$, population size $=40$, mutation function $=$ Gaussian function, and crossover fraction $=0.8$. The deviation of the friction torque was defined as $e(k)=K_{m} I_{a}(k)-\tilde{M}_{f}(k)-\tilde{J} \dot{\omega}(k)$, where $\tilde{M}_{f}(k)$ and $\tilde{J}$ were the parameters to be identified.

The fitness function was defined as $J=\sqrt{\sum_{i=0}^{n} e(k)^{2}}$, and the target fitness value was set as $0.01 \mathrm{~N} \cdot \mathrm{m}$.

Using the simulation result and a genetic algorithm, after an iterative evolution in the 469th generation, the fitness value reached the target we set. The convergence procedure of the system parameters and system load parameters under the action of the genetic algorithm is shown in Figure 12.

After the selection of the genetic algorithm, the identified parameter values of the system model and the system load model were as shown in Table 1.

Base on the identified results in Table 1, the identification of the parameter in the working load could be carried out.

4.2.2. Case 2: $F_{P}=3000 \mathrm{~N}$ and $\theta_{c}=20 \cdot \sin (0.4 \pi r t)$. The parameter array to be identified was defined as $x=$ $\left[\begin{array}{llll}\mu_{c} & \mu_{s} & \omega_{s L} & \mu_{2}\end{array}\right]$, and the initial configuration of the 
TABLE 1: Original values and identified values of the system load model.

\begin{tabular}{lccccc}
\hline & $M_{c}(\mathrm{~N} \cdot \mathrm{m})$ & $M_{s}(\mathrm{~N} \cdot \mathrm{m})$ & $\omega_{s f}(\mathrm{rad} / \mathrm{s})$ & $\sigma_{2}(\mathrm{~N} \cdot \mathrm{m} \cdot \mathrm{s} / \mathrm{rad})$ & $J\left(\mathrm{~kg} \cdot \mathrm{m}^{2}\right)$ \\
\hline Original value & 6.8250 & 12.025 & 0.0577 & 5.3462 & 0.000204 \\
Identified value & 6.7478 & 11.935 & 0.0577 & 5.5400 & 0.0002048 \\
Error & $1.13 \%$ & $0.74 \%$ & $0 \%$ & $3.62 \%$ & $0.39 \%$ \\
\hline
\end{tabular}
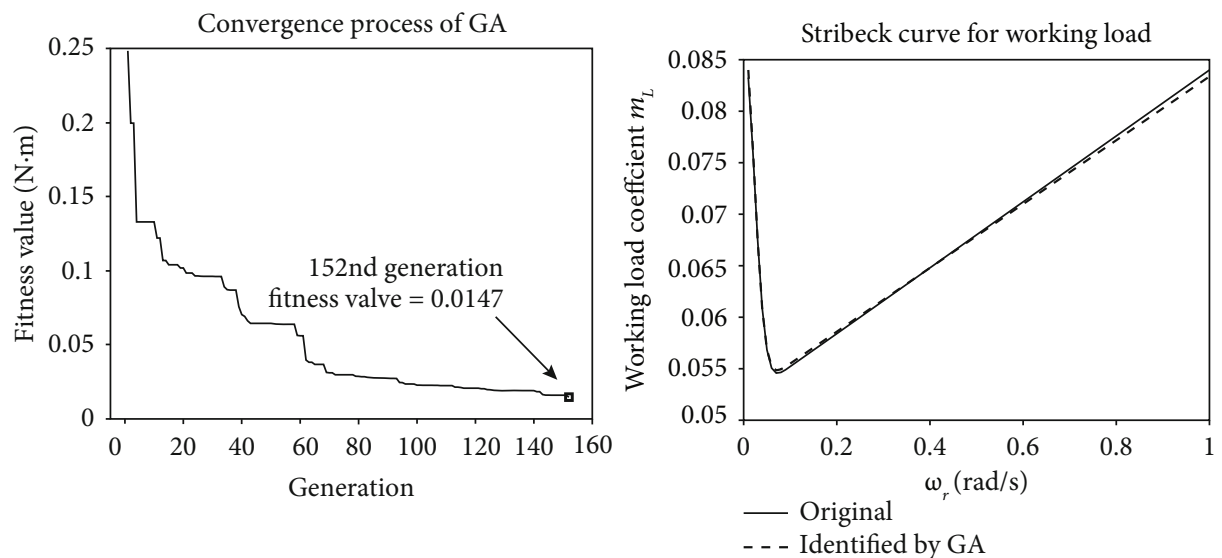

FIgURe 13: Parameter identification result of the working load model using a genetic algorithm.

TABLE 2: Original values and identified values of the working load model.

\begin{tabular}{lcccc}
\hline & $\mu_{c}$ & $\mu_{s}$ & $\omega_{s L}(\mathrm{rad} / \mathrm{s})$ & $\mu_{2}$ \\
\hline Original value & 0.0520 & 0.0860 & 0.0326 & 0.032 \\
Identified value & 0.0524 & 0.0869 & 0.0318 & 0.031 \\
Error & $0.77 \%$ & $1.05 \%$ & $2.45 \%$ & $3.12 \%$ \\
\hline
\end{tabular}

genetic algorithm was the same as that in Case 1. The deviation of the friction torque was defined as $e(k)=K_{m} I_{a}(k)-$ $\widehat{M}_{f}(k)-F_{p} \cdot L_{1} \cdot \tilde{m}_{L}(k)-\widehat{J} \dot{\omega}(k)$, where $\widehat{M}_{f}(k)$ and $\widehat{J}$ used the values that were obtained in Case 1 and $\tilde{m}_{L}(k)$ was the parameter to be identified in this case.

The fitness function was defined as $J=\sqrt{\sum_{i=0}^{n} e(k)^{2}}$, and the target fitness value was set to $0.015 \mathrm{~N} \cdot \mathrm{m}$.

Using the simulation result and a genetic algorithm, after an iterative evolution in the 152nd generation, the fitness value reached the target we set. The convergence procedure of the system parameter and the system load parameter under the action of the genetic algorithm is shown in Figure 13.

After the selection of the genetic algorithm, the identified parameter values of the working load model were as shown in Table 2.

\section{Conclusion}

The load characteristics of the flow regulator for a ducted rocket were examined in this paper, and the loads of the flow regulator were divided into two parts: the system load and the working load. The influences of these loads on the working quality of the flow regulator and ducted rocket were also analyzed. The models of the gas generator, actuator, and load were built based on the working mechanism and the Stribeck friction model. Furthermore, considering the inapplicability of the conventional Stribeck identification method, a new Stribeck parameter identification method for flow regulator was proposed. A sine wave was used as the signal source, and specially designed devices were used to accomplish the switch of the working condition. Using the genetic algorithm, the rotational inertia and parameters of the system load and working load were identified by stages. Finally, the validation of the identification method was carried out with a simulation. More generally, the load characteristics analyzed in this article will contribute to the structure optimization for the flow regulator, and the load modeling methods of the flow regulator in this paper provide valuable tools as a starting point for the compensation control of a ducted rocket.

\section{Data Availability}

The data used to support the findings of this study are available from the corresponding author upon request.

\section{Conflicts of Interest}

The authors declare no conflict of interest. 


\section{Authors' Contributions}

The conceptualization and methodology were handled by A.W. and Q.Z.; the software, validation, and formal analysis by A.W.; the investigation and resources by Q.Z.; the data curation by A.W.; the original draft preparation (writing) by A.W.; the review and editing (writing) by A.W. and Q.Z.; the visualization by A.W.; and the supervision, project administration, and funding acquisition, by Q.Z.

\section{Acknowledgments}

This work was supported by the National Natural Science Foundation of China (Grant No. 61174120).

\section{References}

[1] D. Huo, D. Yan, and B. Gao, "Research progresses and prospect of variable flow ducted rocket technologies," Journal of Solid Rocket Technology, vol. 40, no. 1, pp. 11-19, 2017.

[2] H. Besser, "History of ducted rocket development at BayernChemie," in 44th AIAA/ASME/SAE/ASEE Joint Propulsion Conference \& Exhibit, pp. 21-23, Hartford, CT, USA, July 2008.

[3] Z. Lv, Z. Xia, and B. Liu, "Review of research on solid fuel scramjet engine," Journal of Aerospace Power, vol. 31, no. 8, 2016.

[4] R. A. Stowe, C. Dubois, P. G. Harris, A. E. H. J. Mayer, A. DeChamplain, and S. Ringuette, "Performance prediction of a ducted rocket combustor using a simulated solid fuel," Journal of Propulsion and Power, vol. 20, no. 5, pp. 936-944, 2004.

[5] D. Chen, F. Yao, and S. Chai, "Sliding mode control with observer for PMSM based on Stribeck friction model," in 2014 Seventh International Symposium on Computational Intelligence and Design, pp. 469-472, Hangzhou, China, 2015.

[6] F. Schmid and A. Mielke, "Existence results for a contact problem with varying friction coefficient and nonlinear forces," ZAMM, vol. 87, no. 8-9, pp. 616-631, 2007.

[7] X. Lu and M. M. Khonsari, "An experimental investigation of dimple effect on the Stribeck curve of journal bearings," Tribology Letters, vol. 27, no. 2, article 9217, pp. 169-176, 2007.

[8] O. A. Bauchau, Y. van Weddingen, and S. Agarwal, "Semiactive Coulomb friction lead-lag dampers," Journal of the American Helicopter Society, vol. 55, no. 1, pp. 120051200512, 2010.

[9] L. Marton and B. Lantos, "Modeling, identification, and compensation of stick-slip friction," IEEE Transactions on Industrial Electronics, vol. 54, no. 1, pp. 511-521, 2007.

[10] C. Canudas-de-Wit, "Comments on a new model for control of systems with friction," IEEE Transactions on Automatic Control, vol. 43, no. 8, pp. 1189-1190, 1998.

[11] W. Tan, X. Li, H. Xiang, J. Zhu, and C. Zhang, "Parameter identification of LuGre model based on analysis of steady state error," Optics and Precision Engineering, vol. 19, no. 3, pp. 664-671, 2011.

[12] Y. Guo, Y. Fu, and P. Zhang, "A novel parameters identification method for LuGre friction model," Machine Tool \& Hydraulics, vol. 43, no. 1, pp. 149-153, 2015.

[13] D. Shao, S. Xu, and A. Du, "Dynamic friction modelling and parameter identification for electromagnetic valve actuator,"
Journal of Central South University, vol. 25, no. 12, pp. 30043020, 2018.

[14] W. Yu, J. Ma, J. Li, and J. Xiao, "Friction parameter identification and friction compensation for precision servo turning table," Optics and Precision Engineering, vol. 19, no. 11, pp. 2736-2743, 2011.

[15] Q. Liang, J. Zhang, and Y. Wang, "The friction model parameters identification of servo system based on genetic algorithm," Instrumentation Technology, vol. 6, pp. 34-36, 2011.

[16] J. Chang, B. Li, W. Bao, W. Niu, and D. Yu, "Frictioncompensation control of gas-flow regulation for ducted rockets based on adaptive dither method," Journal of Aerospace Engineering, vol. 26, no. 4, pp. 715-720, 2013.

[17] H. Wang, Z. Wang, M. Sun, and H. Wu, "Combustion modes of hydrogen jet combustion in a cavity-based supersonic combustor," International Journal of Hydrogen Energy, vol. 38, no. 27, pp. 12078-12089, 2013.

[18] W. Huang, W. Liu, S. Li, Z. X. Xia, J. Liu, and Z. G. Wang, "Influences of the turbulence model and the slot width on the transverse slot injection flow field in supersonic flows," Acta Astronautica, vol. 73, pp. 1-9, 2012.

[19] Q. Fang, H. Sun, and W. Liu, "Study on the stribeck curve of slider bearing lubrication state under different load," Journal of Mechanical Transmission, vol. 1, pp. 124-126, 2016. 


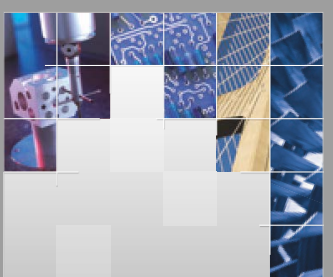

\section{Enfincering}
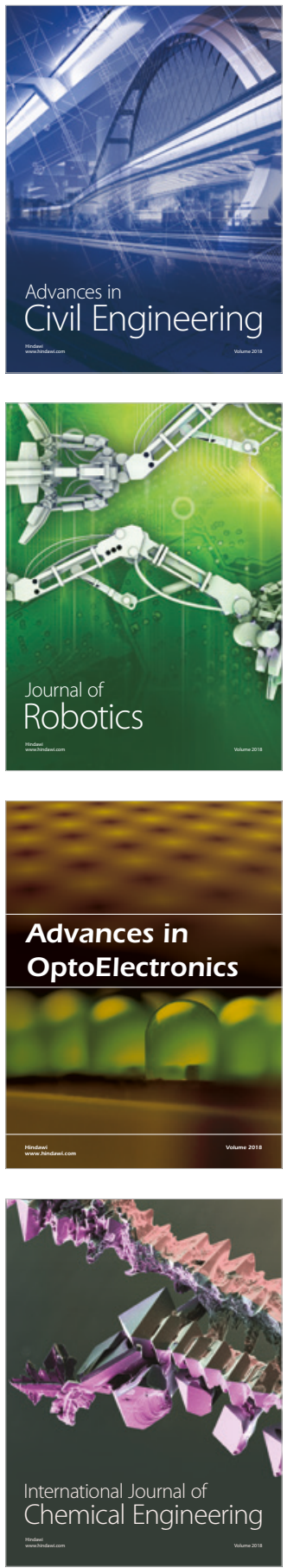

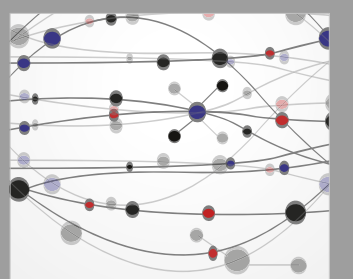

\section{Rotating \\ Machinery}

The Scientific World Journal

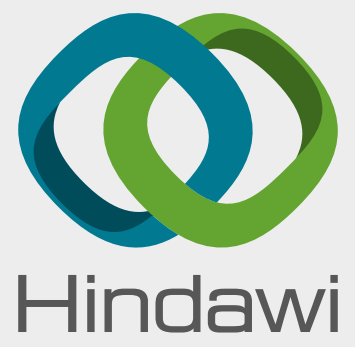

Submit your manuscripts at

www.hindawi.com
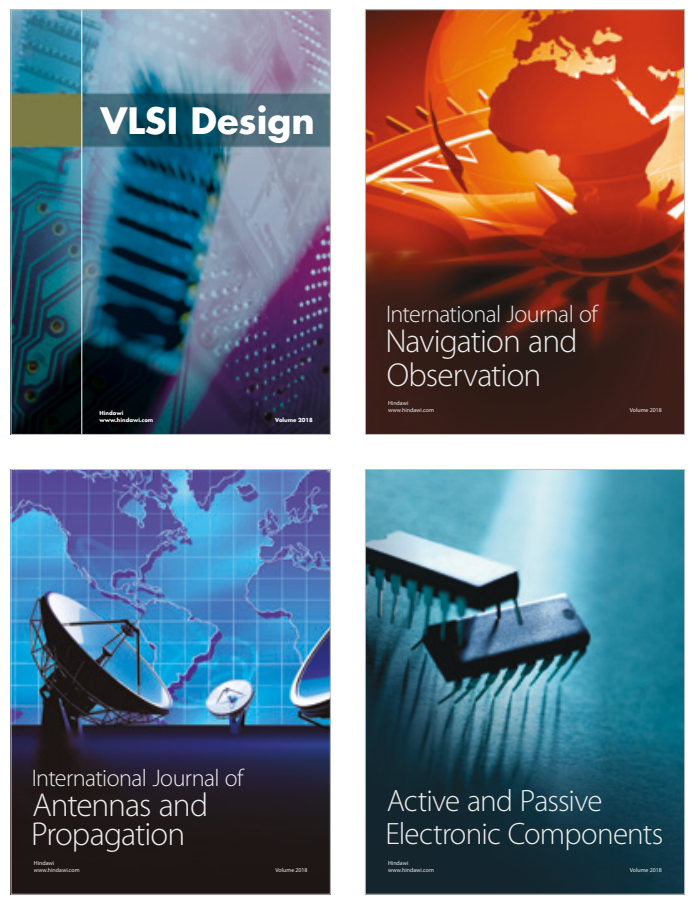
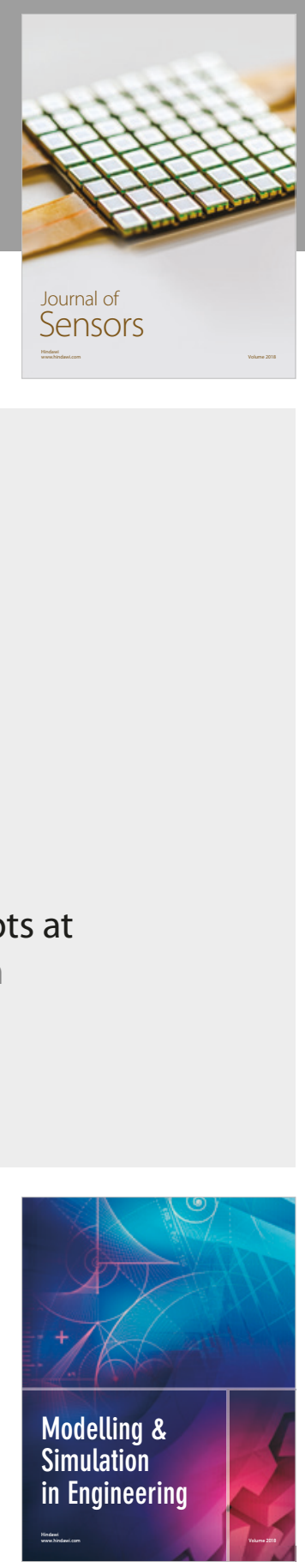

\section{Advances \\ Multimedia}
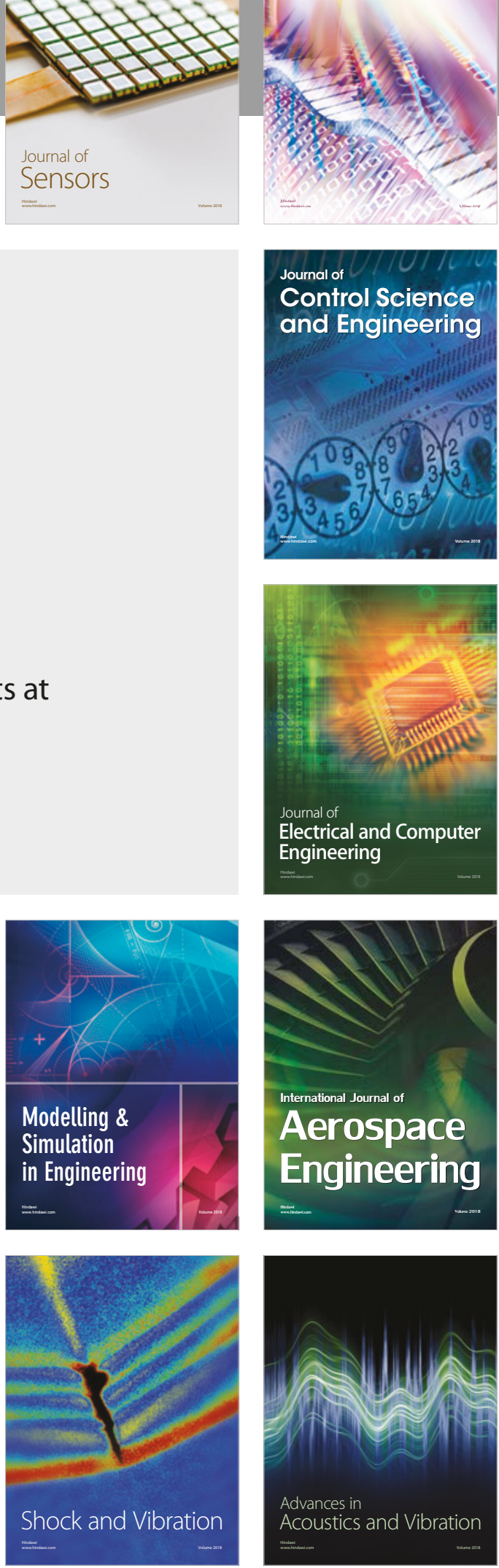